\title{
Development and evaluation of a loop-mediated isothermal amplification assay for rapid and simple detection of Campylobacter jejuni and Campylobacter coli
}

Correspondence
Wataru Yamazaki
wataru@iph.pref.osaka.jp

Received 11 October 2007

Accepted 4 January 2008

\author{
Wataru Yamazaki, ${ }^{1}$ Masumi Taguchi, ${ }^{1}$ Masanori Ishibashi, ${ }^{1}$ \\ Miyoshi Kitazato, ${ }^{2}$ Masafumi Nukina, ${ }^{3}$ Naoaki Misawa ${ }^{4}$ and Kiyoshi Inoue ${ }^{1}$ \\ ${ }^{1}$ Division of Bacteriology, Osaka Prefectural Institute of Public Health, Osaka, Japan \\ ${ }^{2}$ Yodogawa Christian Hospital, Osaka, Japan \\ ${ }^{3}$ Microbiological Department, Kobe Institute of Health, Hyogo, Japan \\ ${ }^{4}$ Department of Veterinary Public Health, Faculty of Agriculture, University of Miyazaki, Miyazaki, \\ Japan
}

\begin{abstract}
We developed a loop-mediated isothermal amplification (LAMP) assay for the rapid and simple detection of Campylobacter jejuni and Campylobacter coli. The assay provides a specific LAMP product for each of these two species. The assay correctly identified $65 \mathrm{C}$. jejuni and $45 \mathrm{C}$. coli strains, but not 75 non-C. jejunilcoli strains. The sensitivity of the LAMP assay for $C$. jejuni and C. coli in spiked human stool specimens was $5.6 \times 10^{3}$ c.f.u. $\mathrm{g}^{-1}$ (1.4 c.f.u. per test tube) and $4.8 \times 10^{3}$ c.f.u. $\mathrm{g}^{-1}$ (1.2 c.f.u. per test tube), respectively. When 90 stool specimens from patients with diarrhoea were tested by LAMP and direct plating, the LAMP results showed $81.3 \%$ sensitivity and $96.6 \%$ specificity compared to isolation of $C$. jejuni and C. coli by direct plating. Further, the LAMP assay required less than $2 \mathrm{~h}$ for detection of $C$. jejuni and C. coli in stool specimens. This LAMP assay is a rapid and simple tool for the detection of C. jejuni and C. coli and will be useful in facilitating the early diagnosis of food poisoning incidents caused by these organisms.
\end{abstract}

\section{INTRODUCTION}

Campylobacter is widely acknowledged as one of the most frequent causes of acute bacterial gastroenteritis in humans worldwide. Campylobacter jejuni and Campylobacter coli are the predominant cause of campylobacteriosis (Lawson et al., 1999; Nachamkin, 2003; On, 2005). C. jejuni and C. coli diarrhoea in humans is commonly diagnosed by isolating these pathogens from a stool specimen by a bacterial culture test. The test procedure includes the plating of stool onto selective agar medium and subsequent identification of suspected colonies on the agar medium. The bacterial culture tests require at least 4 days and are time-consuming and laborious due to the fastidious and slow growth of these species. A rapid, simple and practical assay for the identification of $C$. jejuni and $C$. coli has thus been sought.

Several PCR assays offer a more sophisticated approach to the identification of Campylobacter species (Linton et al., 1996; Yamazaki-Matsune et al., 2007). Although PCR assays provide more rapid identification of $C$. jejuni and C. coli than conventional biochemical-based assays, they

Abbreviation: LAMP, loop-mediated isothermal amplification. require the use of electrophoresis to detect amplified products, which is time-consuming and tedious. Further, another real-time PCR assay recently developed for the rapid identification of Campylobacter species (Logan et al., 2001; Sails et al., 2003; Waage et al., 1999) is not routinely used due to the requirement for an expensive thermal cycler with a fluorescence detector.

Among other techniques, one promising candidate is a novel nucleic acid amplification method termed loopmediated isothermal amplification (LAMP; Notomi et al., 2000). LAMP is based on the principle of autocycling strand displacement DNA synthesis performed by the Bst DNA polymerase large fragment for the detection of a specific DNA sequence with specific characteristics, which offers a number of advantages. First, all reactions can be carried out under isothermal conditions ranging from 60 to $65{ }^{\circ} \mathrm{C}$. Second, its use of six primers recognizing eight distinct regions on the target nucleotides means that specificity is extremely high (Nagamine et al., 2002). Third, detection is simplified by visual judgment by the unaided eye without electrophoresis (Hara-Kudo et al., 2005; Song et al., 2005). Thus a LAMP assay is faster and easier to perform than PCR assays, as well as being more specific 
(Hara-Kudo et al., 2007). Furthermore, because the LAMP assay synthesizes a large amount of DNA, the products can be detected by simple turbidity. Thus, compared to PCR assays, expensive equipment is not necessary to give a high level of precision (Hara-Kudo et al., 2005). These features allow simple, rapid and cost-effective detection (Iwamoto et al., 2003; Hara-Kudo et al., 2005). Also, the increase in the turbidity of the reaction mixture according to the production of precipitate correlates with the amount of DNA synthesized (Hara-Kudo et al., 2007). A commercial LAMP assay kit for detection of $C$. jejuni/coli is, however, handicapped by the inability to discern between $C$. jejuni and C. coli. Although various LAMP assays for the identification of pathogenic organisms have been developed (Hara-Kudo et al., 2007; Minami et al., 2006; Ohtsuka et al., 2005; Song et al., 2005), direct detection from clinical stool specimens has not been described.

Here, we describe a rapid and simple LAMP assay for the detection of C. jejuni and C. coli. We evaluated the efficacy of this LAMP assay in the direct detection of 90 human stool specimens obtained from food poisoning incidents.

\section{METHODS}

Bacterial strains. One hundred and eighty-five bacterial strains were used: these included 65 C. jejuni and 45 C. coli strains and an additional 45 Campylobacter and 30 non-Campylobacter strains that were used as reference strains and field isolates. Details of the 155 Campylobacter strains are as follows (a superscript $\mathrm{T}$ designates a type strain). Nineteen Campylobacter reference strains were obtained from international culture collections: Campylobacter coli JCM $2529^{\mathrm{T}}$ (Japan Collection of Microorganisms); Campylobacter fetus subsp. fetus ATCC $27374^{\mathrm{T}}$ (American Type Culture Collection), GTC 08732 (Gifu Type Culture Collection, Gifu, Japan), GTC 08746, GTC 11236 and GTC 12267; Campylobacter fetus subsp. venerealis JCM 2528 ${ }^{\mathrm{T}}$; Campylobacter helveticus ATCC $51209^{\mathrm{T}}$; Campylobacter hyointestinalis subsp. hyointestinalis ATCC $35217^{\mathrm{T}}$; Campylobacter hyointestinalis subsp. lawsonii CCIPH-1 (Culture Collection, Institute of Public Health, Osaka, Japan); Campylobacter jejuni subsp. doylei LMG $8843^{\mathrm{T}}$ (Culture Collection of the Laboratorium voor Microbiologie, University of Ghent); Campylobacter jejuni subsp. jejuni LMG $8841^{\mathrm{T}}$, ATCC 33291, ATCC 33292 and JCM 2013; Campylobacter lari JCM 2530 ${ }^{\mathrm{T}}$; Campylobacter mucosalis ATCC $43264^{\mathrm{T}}$; Campylobacter sputorum ATCC $35980^{\mathrm{T}}$; and Campylobacter upsaliensis ATCC $43954^{\mathrm{T}}$. One hundred and thirty-six Campylobacter isolates were obtained from clinical and environmental sources between 1988 and 2007 in Hyogo, Miyazaki and Osaka, Japan, as follows: $60 \mathrm{C}$. jejuni subsp. jejuni isolates from clinical sources $(n=34)$, poultry $(n=18)$, seagull faeces $(n=7)$ and puppy faeces $(n=1)$; 44 C. coli isolates from clinical sources $(n=36)$, poultry $(n=5)$ and pigs $(n=3) ; 9$ C. hyointestinalis subsp. hyointestinalis from simian faeces $(n=5)$, cattle faeces $(n=3)$ and turtle faeces $(n=1) ; 9$ C. lari isolates from seagull faeces $(n=8)$ and an unknown source $(n=1) ; 9$ C. upsaliensis isolates from adult dog faeces $(n=6)$ and puppy faeces $(n=3)$; and 5 C. fetus subsp. fetus isolates from clinical sources $(n=4)$ and cattle liver $(n=1)$.

Seven non-Campylobacter reference strains were obtained from international culture collections (Arcobacter butzleri ATCC $49616^{\mathrm{T}}$; Arcobacter cryaerophilus ATCC $43158^{\mathrm{T}}$; Arcobacter skirrowii ATCC $51132^{\mathrm{T}}$; Escherichia coli ATCC 25922 and ATCC 35218; Pseudomonas aeruginosa ATCC 27853; and Staphylococcus aureus subsp. aureus ATCC 25923). Twenty-three non-Campylobacter isolates were obtained from clinical sources (five Helicobacter pylori isolates; and one isolate each of Aeromonas hydrophila, Aeromonas sobria, Citrobacter freundii, Enterobacter cloacae, enterotoxigenic Escherichia coli O169: H41 heatlabile enterotoxin-positive, enterotoxigenic Escherichia coli O114: $\mathrm{H}-$ heat-labile enterotoxin- and heat-stable enterotoxin-positive, Klebsiella pneumoniae, Morganella morganii, Plesiomonas shigelloides, Proteus mirabilis, Providencia alcalifaciens, Salmonella enterica serovar Enteritidis, Shigella flexneri 1a, Shigella sonnei, Vibrio cholerae O1 Ogawa cholera toxin-positive, Vibrio fluvialis, Vibrio mimicus and Vibrio parahaemolyticus $\mathrm{O} 3$ : K6 thermostable direct haemolysin-positive).

Storage and culture conditions. All Campylobacter, Arcobacter and Helicobacter strains were stored in brucella broth containing $10 \%$ $(\mathrm{v} / \mathrm{v})$ horse serum and $10 \%(\mathrm{v} / \mathrm{v})$ DMSO at $-80{ }^{\circ} \mathrm{C}$ until use. They were grown on blood agar supplemented with $5 \%(\mathrm{v} / \mathrm{v})$ lysed horse blood and incubated for 2-3 days in a microaerobic atmosphere, except H. pylori, which was incubated for 7-10 days. Microaerobic conditions were generated with an AnaeroPack MicroAero (Mitsubishi Gas Chemical), which maintained an atmosphere of approximately $8 \% \mathrm{O}_{2}, 7 \% \mathrm{CO}_{2}$ and $85 \% \mathrm{~N}_{2}$. All strains were grown at $37{ }^{\circ} \mathrm{C}$, except for Arcobacter cryaerophilus, which was grown at $30{ }^{\circ} \mathrm{C}$. Other bacterial strains were stored in cooked meat broth at room temperature until use, and grown on blood agar and cultured overnight at $37{ }^{\circ} \mathrm{C}$ under aerobic conditions.

DNA extraction from culture. Bacterial DNA was extracted as previously described (Misawa et al., 2002; Yamazaki-Matsune et al., 2007). A single loopful of culture was inoculated in $50 \mu \mathrm{l} \mathrm{NaOH}$ $\left(25 \mathrm{mmol} \mathrm{l}^{-1}\right)$ in a $1.5 \mathrm{ml}$ microcentrifuge tube using a disposable loop ( $1 \mathrm{~mm}$ diameter), and the cell mixture was heated at $100{ }^{\circ} \mathrm{C}$ for $10 \mathrm{~min}$. After neutralization with $50 \mu \mathrm{l}$ Tris/ $\mathrm{HCl}$ buffer $(80 \mathrm{mmol}$ $\left.1^{-1}, \mathrm{pH} 7.5\right)$, cell debris was pelleted by centrifugation at $20000 \mathrm{~g}$ and $4{ }^{\circ} \mathrm{C}$ for $5 \mathrm{~min}$ and the supernatant was used as template DNA for LAMP and multiplex PCR assays.

Identification of Campylobacter strains. Campylobacter strains were identified with biochemical-based and multiplex PCR assays according to a previously described method (Yamazaki-Matsune et al., 2007).

LAMP assay. The LAMP assay was performed with a Loopamp DNA amplification kit (Eiken Chemical). The final LAMP assay comprised $5 \mu \mathrm{l}$ template DNA, $1 \mu \mathrm{l}$ Bst DNA polymerase (Eiken Chemical), $1.6 \mu \mathrm{mol} \mathrm{l}^{-1}$ each of inner primers FIP and BIP, $0.2 \mu \mathrm{mol} 1^{-1}$ each of outer primers $\mathrm{F} 3$ and $\mathrm{B} 3$, and $0.8 \mu \mathrm{mol} 1^{-1}$ each of loop primers LoopF and LoopB, in $1 \times$ Reaction Mix (Eiken Chemical). The final volume was adjusted to $25 \mu \mathrm{l}$. All primers were produced by Hokkaido System Science, and were designed from the sequence data submitted to GenBank (AL111168, cj0414 for C. jejuni; and AAFL01000003, CCO0367 for C. coli) with Primer Explorer V4 software (Fujitsu System Solutions). The sequences and locations of each primer are shown in Table 1 and Fig. 1. Primer FIP consisted of the F1 complementary sequence and the F2 sequence. Primer BIP consisted of the B1 direct sequence and the B2 complementary sequence. Primers B3 and LF consisted of the B3 and LF complementary sequences, respectively. The mixture was incubated at $65{ }^{\circ} \mathrm{C}$ for $60 \mathrm{~min}$ and then at $80{ }^{\circ} \mathrm{C}$ for $2 \mathrm{~min}$ to terminate the reaction in a Loopamp real-time turbidimeter (LA-320; Teramecs). LAMP amplification was detected as the turbidity at $650 \mathrm{~nm}$ using a LA-320 turbidimeter in real-time. The reaction was considered to be positive when the turbidity reached 0.1 within $60 \mathrm{~min}$. Turbidity visible with the unaided eye was also considered to indicate a successful LAMP procedure.

Determination of the sensitivity of the LAMP assay. To determine the sensitivity of the LAMP assay for the detection of C. jejuni and C. coli in human stool specimens, known amounts of 
Table 1. LAMP primers used

\begin{tabular}{|c|c|c|c|c|}
\hline Target gene & $\begin{array}{c}\text { GenBank } \\
\text { accession no. }\end{array}$ & Primer & Sequence $\left(5^{\prime} \rightarrow 3^{\prime}\right)$ & Gene location (bp) \\
\hline \multirow[t]{6}{*}{ cj0414 } & AL111168 & CJ-FIP & $\begin{array}{l}\text { ACAGCACCGCCACCTATAGT- } \\
\text { AGAAGCTTTTTTAAACTAGGGC (Flc-F2) }\end{array}$ & 95-76 (Flc), 25-46 (F2) \\
\hline & & CJ-BIP & $\begin{array}{l}\text { AGGCAGCAGAACTTACGCATT- } \\
\text { GAGTTTGAAAAAACATTCTACCTCT (B1-B2c) }\end{array}$ & $101-121$ (B1), 181-157 (B2c) \\
\hline & & CJ-F3 & GCAAGACAATATTATTGATCGC (F3) & $3-24$ \\
\hline & & CJ-B3 & CTTTCACAGGCTGCACTT (B3c) & $218-201$ \\
\hline & & CJ-LF & CTAGCTGCTACTACAGAACCAC (LFc) & $74-53$ \\
\hline & & CJ-LB & CATCAAGCTTCACAAGGAAA (LB) & $124-143$ \\
\hline \multirow[t]{6}{*}{$\mathrm{CCO} 0367 \dagger$} & AAFL01000003 & CC-FIP & $\begin{array}{l}\text { AAGAGATAAACACCATGATCCCAG- } \\
\text { TCATGAATGAGCTTACTTTAGC (Flc-F2) }\end{array}$ & 730-707 (Flc), 665-686 (F2) \\
\hline & & CC-BIP & $\begin{array}{l}\text { GCGGCAAAGACTTATGATAAAGC- } \\
\text { TACCGCCATTCCTAAAACAAG (B1-B2c) }\end{array}$ & $748-770$ (B1), 810-790 (B2c) \\
\hline & & CC-F3 & TGGGAGCGTTTTTGATCT (F3) & $641-658$ \\
\hline & & CC-B3 & AATCAAACTCACCGCCAT (B3c) & $828-811$ \\
\hline & & CC-LF & CCACTACAGCAAAGGTGATG (LFc) & $706-687$ \\
\hline & & CC-LB & CCACGATAGCCTTTATGGA (LB) & $771-789$ \\
\hline
\end{tabular}

${ }^{\star}$ Presumed to encode an oxidoreductase from the sequence of AL111168, submitted to GenBank by Parkhill et al. (2000). $\dagger$ Presumed to encode a gufA gene from the sequence of AAFL01000003, submitted to GenBank by Fouts et al. (2005).

C. jejuni LMG $8841^{\mathrm{T}}$ and C. coli JCM $2529^{\mathrm{T}}$ were used. Cultures were incubated at $37^{\circ} \mathrm{C}$ for $48 \mathrm{~h}$ in a microaerobic atmosphere. Serial 10 fold dilutions of the culture were prepared in PBS and $100 \mu \mathrm{l}$ of each was spiked into $100 \mathrm{mg}$ of a Campylobacter-negative stool specimen, and then each of the faecal homogenates was adjusted to a $10 \%$ concentration with PBS. After mixing well, $100 \mu$ of each was centrifuged for $5 \mathrm{~min}$ at $20000 \mathrm{~g}$ in a $1.5 \mathrm{ml}$ microcentrifuge tube.
After removal of the supernatant, the pellets were resuspended in $100 \mu \mathrm{l} \mathrm{NaOH}\left(25 \mathrm{mmol} \mathrm{l}^{-1}\right)$, and the mixture was heated at $100{ }^{\circ} \mathrm{C}$ for $10 \mathrm{~min}$. After neutralization with $100 \mu \mathrm{l}$ Tris/HCl buffer (80 $\mathrm{mmol}^{-1}, \mathrm{pH} \mathrm{7.5),} \mathrm{debris} \mathrm{was} \mathrm{pelleted} \mathrm{by} \mathrm{centrifugation} \mathrm{at}$ $20000 \mathrm{~g}$ and $4{ }^{\circ} \mathrm{C}$ for $5 \mathrm{~min}$. Five microlitres of each supernatant was then used as template DNA for the LAMP assay. Spiked cells in a stool specimen were diluted 1:4000 in LAMP reaction tubes. The

(a)

1 ATGGAAGACAATATAATGATCGCAGAAGC I T TTAAA I T AG GGCTTCTAGGTGGTICT F3 F2

61 GTAGTAGCAGCTAGCACTATAGGTGGCGGTGCTGTTTTAAAGGCAGCAGAACTTACGCAT LF $\quad$ F1

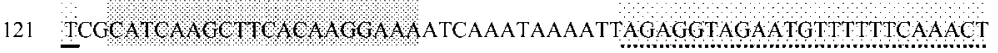
LB B2

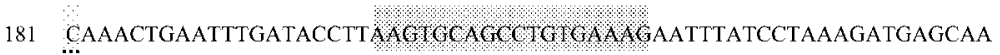
B3

(b)

601 TCTGCACTTTCGGGTGTAGCCGAGCCTATGGGAGCGATTGIGGGAGCGTTTTTGATCTTG F3

661 CCTATCATGAATGAGCT ACTTTAGCCATCACCHTGCTGTAGTGGCTGGGATCATGGTG F2 $\mathrm{LF}$

721

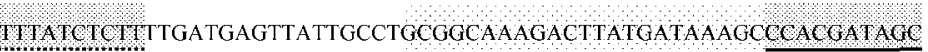
B1

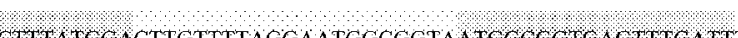

781 CTMATGGACTGTTTAGGAATGGCGGTAA TGGEGC KGAGTITGATI $\overline{\mathrm{B}}$ $\mathrm{B} 2$ B3
Fig. 1. Locations of the target sequences used as primers. The name and location of each target sequence as a primer in $C$. jejuni cj0414 and C. coli CCO0367 are shown in (a) and (b), respectively. 
sensitivity tests were conducted in triplicate, and the detection limits were defined as the last positive dilutions, with the sample considered positive if all three samples tested positive. In parallel, to enumerate the bacteria, $100 \mu \mathrm{l}$ aliquots of appropriate dilutions were spread on blood agar and incubated at $37^{\circ} \mathrm{C}$ in a microaerobic atmosphere. Colonies were counted at the dilution yielding 20-200 c.f.u. after 5 days, and c.f.u. ( $\mathrm{ml}$ suspension $)^{-1}$ was calculated.

Preparation of human stool specimens. A total of 90 stool specimens were obtained from 90 patients with diarrhoea derived from 22 different food poisoning incidents that occurred in Osaka Prefecture, Japan, between November 2006 and September 2007. The number of patients of individual food poisoning incidents ranged from 2 to 11. Cases were included for analysis if stool specimens were collected within 7 days from symptom onset. The specimens, all of which were diarrhoeal stool specimens, were transported to our laboratory from 13 local public health centres in Osaka Prefecture and subjected to microbiological examination for enteropathogens, including C. jejuni and C. coli, within $6 \mathrm{~h}$ of arrival at our laboratory. The causative agent of individual food poisoning incidents was determined according to the results of a microbiological examination. The incidents which led to the isolation of C. jejuni/coli were designated C. jejuni/coli food poisoning and the incidents which led to the isolation of non-C. jejuni/coli enteropathogens were designated non-C. jejuni/coli food poisoning. Of the 22 incidents, 13 were $C$. jejunilcoli food poisoning and 9 were non-C. jejuni/coli food poisoning caused by non-C. jejuni/coli enteropathogens. For the isolation of C. jejuni and C. coli, approximately one loopful of stool specimen was streaked onto Skirrow agar (Oxoid) and/or modified charcoal cefoperazone deoxycholate agar (mCCDA; Oxoid) using a disposable loop ( $10 \mathrm{~mm}$ diameter), and the plates were incubated at $42{ }^{\circ} \mathrm{C}$ for $48 \mathrm{~h}$. Two typical campylobacter-like colonies were selected and subcultured at $42{ }^{\circ} \mathrm{C}$ for $48 \mathrm{~h}$ for identification. In parallel, for LAMP testing of the direct detection of $C$. jejuni and C. coli from faecal stool specimens, each stool specimen was adjusted to a $10 \%$ homogenate with PBS and stored at $-20{ }^{\circ} \mathrm{C}$ until use, at which time $100 \mu \mathrm{l}$ of each was used for preparation of DNA templates as described above, and the total volume was adjusted to $200 \mu$ l. Five microlitres of each was used for the LAMP assay. The LAMP assay was also carried out on the cultured C. jejuni and C. coli strains from the stool specimen to check the specificity of the LAMP reaction.

\section{RESULTS AND DISCUSSION}

Here, we report a novel and highly specific LAMP assay for the identification of $C$. jejuni and C. coli. This assay provides markedly more simple and rapid detection of C. jejuni/coli than conventional biochemical-based and PCR assays. Further, it can be applied to the direct detection of C. jejuni/coli in stool specimens.

A commercial LAMP assay kit for the detection of $C$. jejunil coli is available (Furuhata et al., 2006). However, this kit is unable to discern between $C$. jejuni and C. coli, and the sequences of its primer sets are not public. We therefore developed a new LAMP assay for C. jejuni and C. coli. A C. jejuni primer set based on the putative oxidoreductase gene (Wang et al., 1992; Parkhill et al., 2000) was designed, on the basis of the excellent specificity of this gene for C. jejuni in our previous study (Yamazaki-Matsune et al., 2007). A C. coli primer set based on the putative gufA (heavy metal cations transporter; Asakura et al., 2007; Fouts et al., 2005) gene was designed since the sequence of this gene has proven to be highly conserved and specific for C. coli (Asakura et al., 2007).

LAMP products were obtained from all 65 C. jejuni and 45 C. coli strains (Table 2). No LAMP products were obtained from any of the 75 non-C. jejuni/ coli strains. The sensitivity of the LAMP assay for C. jejuni LMG $8841^{\mathrm{T}}$ and C. coli JCM $2529^{\mathrm{T}}$ in spiked human stool specimens was found to be $5.6 \times 10^{3}$ c.f.u. $\mathrm{g}^{-1}$ (1.4 c.f.u. per test tube) and $4.8 \times 10^{3}$ c.f.u. $\mathrm{g}^{-1}$ (1.2 c.f.u. per test tube), respectively. The LAMP product showed an increase in turbidity (Fig. 2) and was visible as white turbidity. Sensitivities determined by the two methods were constantly matched with each other.

To evaluate the efficacy of LAMP assay testing of stool specimens for the diagnosis of C. jejuni/coli diarrhoea, 90 stool specimens from diarrhoea patients in food poisoning

Table 2. Results of LAMP assay

\begin{tabular}{|lccc|}
\hline Strain & No. of strains tested & \multicolumn{2}{c|}{ Positive number by LAMP } \\
\cline { 2 - 4 } & & C. jejuni & C. coli \\
\hline C. jejuni subsp. jejuni & 64 & 64 & 0 \\
C. jejuni subsp. doylei & 1 & 1 & 0 \\
C. coli & 45 & 0 & 45 \\
C. fetus subsp. fetus & 10 & 0 & 0 \\
C. fetus subsp. venerealis & 1 & 0 & 0 \\
C. hyointestinalis subsp. hyointestinalis & 10 & 0 & 0 \\
C. hyointestinalis subsp. lawsonii & 1 & 0 & 0 \\
C. lari & 10 & 0 & 0 \\
C. upsaliensis & 10 & 0 & 0 \\
C. helveticus & 1 & 0 & 0 \\
C. mucosalis & 1 & 0 & 0 \\
C. sputorum & 1 & 0 & 0 \\
Non-Campylobacter bacteria & 30 & 0 & 0 \\
\hline
\end{tabular}



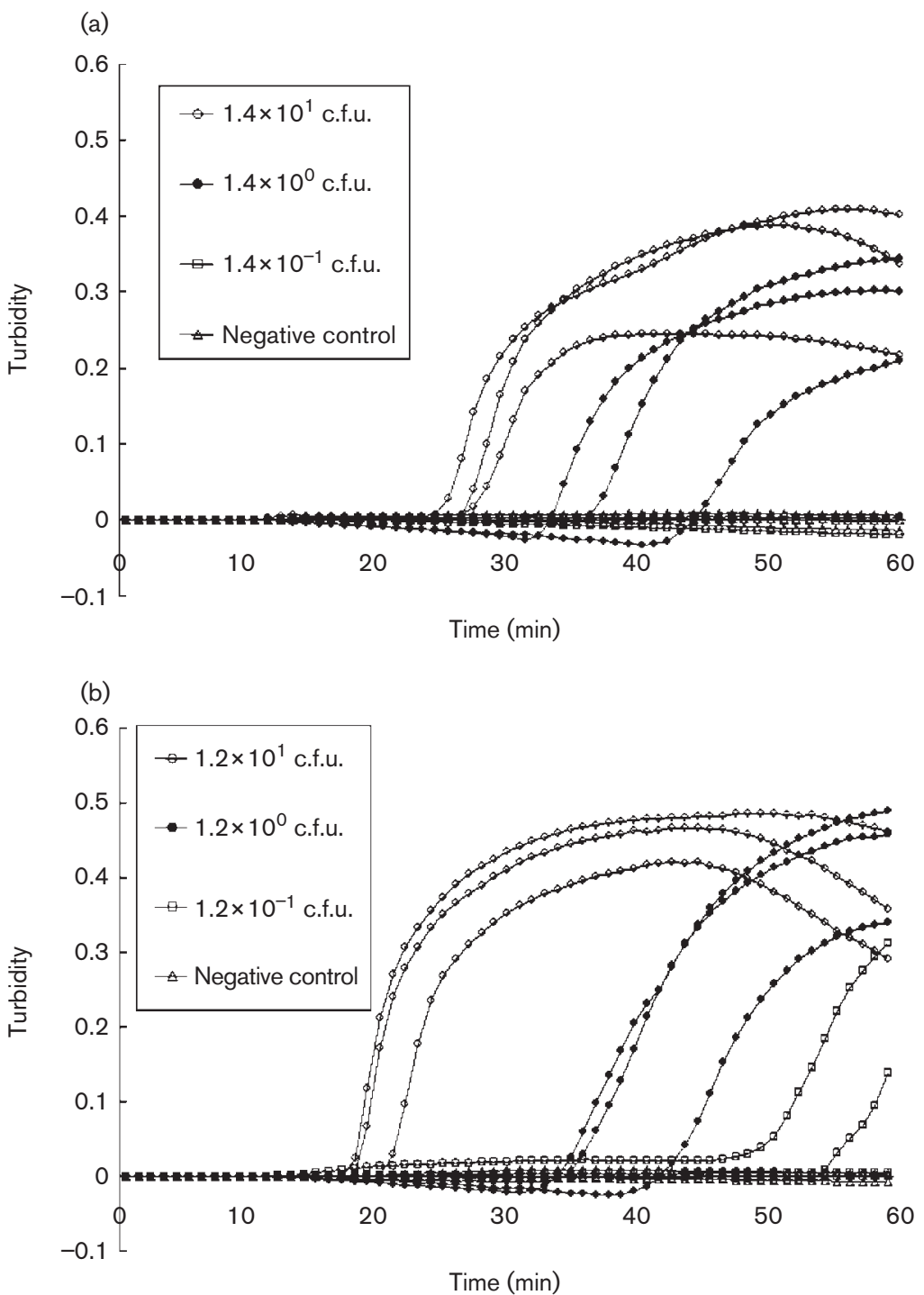

Fig. 2. Sensitivity test to detect $C$. jejuni and C. coli using a real-time turbidimeter. The curves from left to right indicate decreasing numbers of bacterial colonies [14-0.14 c.f.u. per test tube in (a) and 12-0.12 c.f.u. per test tube in (b)]. (a) Detection of C. jejuni; (b) detection of C. coli. incidents were tested with the LAMP assay. The LAMP results were compared with the results of a direct plating culture test for C. jejuni/coli. The results are summarized in Table 3. C. jejuni and C. coli were isolated from 29 and 3 of the 90 stool specimens, respectively. Of the 32 C. jejuni/coli culture stool specimens (29 C. jejuni and 3 C. coli) positive by direct plating, 26 were positive by LAMP assay. Of these 26 LAMP-positive specimens, 21 were $C$. jejuni-positive and 5 were both C. jejuni- and C. coli-positive. Of the remaining $58 \mathrm{C}$. jejuni/coli culture stool specimens negative by direct plating, 2 and 56 stool specimens yielded $C$. jejuni-positive and C. jejuni/coli-negative results by LAMP assay, respectively. The LAMP assay therefore showed $81.3 \%$ sensitivity (26 of 32 specimens) and $96.6 \%$ specificity (56 of 58 specimens) compared with the isolation of $C$. jejuni/coli by the conventional direct plating culture test. Further, the LAMP assay correctly identified the 29 C. jejuni and 3 C. coli strains isolated from stool specimens. Of 32 C. jejuni/coli culture-positive specimens,
2 and 1 were co-isolated enterohaemorrhagic Escherichia coli $\mathrm{O} 157$ verotoxin 2-positive and Norovirus, respectively. Among the $58 \mathrm{C}$. jejuni/coli culture-negative specimens, 14, 6, 4 and 2 were isolated Salmonella spp., Norovirus, Clostridium perfringens enterotoxin A-positive and $V$. parahaemolyticus $\mathrm{O} 3$ : $\mathrm{K} 6$ thermostable direct haemolysinpositive, respectively. The bacterial culture test for the isolation and identification of C. jejunilcoli from the stool specimens required 4 days, with direct plating onto selective agars and sequential subculture. In contrast, the LAMP assay was markedly faster, requiring less than $2 \mathrm{~h}$ from the beginning of DNA extraction to final determination for the direct detection of human stool specimens.

Six culture-positive/LAMP-negative specimens were obtained as shown in Table 3. The cause of this discrepancy may have been the small number of C. jejunilcoli in the stool specimens, presumed lower than $4.8-5.6 \times 10^{3}$ c.f.u. $\mathrm{g}^{-1}$, which was insufficient for LAMP detection. In a preliminary 
Table 3. Comparison of results for LAMP and direct plating of C. jejuni and C. coli from 90 stool specimens

\begin{tabular}{|c|c|c|c|c|c|}
\hline \multirow[t]{2}{*}{ No. of specimens } & \multicolumn{2}{|c|}{ Results of direct plating* } & \multicolumn{2}{|c|}{ LAMP results ${ }^{*}$} & \multirow[t]{2}{*}{ Source of specimens } \\
\hline & C. jejuni & C. coli & C. jejuni & C. coli & \\
\hline 3 & $0 / 3$ & $3 / 3$ & $3 / 3$ & $3 / 3$ & Patients with $C$. jejuni/coli food poisoning \\
\hline 2 & $2 / 2$ & $0 / 2$ & $2 / 2$ & $2 / 2$ & Patients with C. jejuni/coli food poisoning \\
\hline 6 & $6 / 6$ & $0 / 6$ & $0 / 6$ & $0 / 6$ & Patients with C. jejuni/coli food poisoning \\
\hline $32 \S$ & $0 / 32$ & $0 / 32$ & $0 / 32$ & $0 / 32$ & Patients with non-C. jejuni/coli food poisoning \\
\hline
\end{tabular}

${ }^{*}$ Number positive/number of specimens tested.

†Of 21 specimens, enterohaemorrhagic Escherichia coli O157 verotoxin 2-positive and Norovirus were co-isolated from 2 and 1, respectively. ¥Of 24 specimens, Norovirus and Salmonella spp. were isolated from 4 and 1, respectively.

§Of 32 specimens, Salmonella spp., Clostridium perfringens enterotoxin A-positive, Norovirus and Vibrio parahaemolyticus O3 : K6 thermostable direct haemolysin-positive were isolated from $13,4,2$ and 2, respectively.

study, a LAMP assay was carried out on 10- and 100-fold diluted supernatants from the six culture-positive/LAMPnegative specimens to exclude the influence of inhibitory factors in the stool specimens (Hoshino et al., 1998; Persson \& Olsen, 2005; Ramamurthy et al., 1993). The results were all negative, which may suggest that the number of $C$. jejuni/coli in the six stool specimens was small.

Of 58 specimens obtained from patients of $C$. jejuni/coli food poisoning incidents, 32, 2 and 24 specimens were culturepositive, culture-negative/LAMP-positive and culture-negative/LAMP-negative, respectively. All 58 specimens were potential carriers of $C$. jejuni/coli due to the ingestion of C. jejuni/coli-contaminated foods. However, C. jejuni/coli was not isolated from 26 of the 58 patients by direct plating culture, presumably due to any of the influence of a small ingestion amount, natural excretion, medication, rapid loss of cell viability in stools during transportation and storage, small number of cells in stool specimens and false-negative results by the overgrowth of normal flora on selective agars (Kulkarni et al., 2002; Persson \& Olsen, 2005; Ramamurthy et al., 1993; Sails et al., 2003). Of the 26 specimens, 2 were culture-negative/C. jejuni LAMP-positive. The remaining 24 specimens, as well as 32 control specimens obtained from patients of non-C. jejuni/coli food poisoning incidents and unrelated to $C$. jejuni/coli, were culture-negative/LAMPnegative. The cause of this discrepancy was thus presumed not to be false-positives but rather the detection of bacteria in stool specimens that were sufficiently stressed to resist conventional culture under standard conditions or falsenegative results due to the overgrowth of normal flora contained in stool specimens (Kulkarni et al., 2002; Persson \& Olsen, 2005; Shirai et al., 1991; Sails et al., 2003) on selective agars. These results may suggest that the LAMP assay could detect the specific sequences of $C$. jejuni even when C. jejuni was sufficiently stressed to resist conventional culture, but with the chromosomal DNA released by autolysis or the overgrowth of normal flora inhibiting separation of C. jejuni on selective agars. Further work is needed to confirm this hypothesis.

As shown in Table 3, the three C. coli and two C. jejuni culture-positive specimens were both $C$. coli and $C$. jejuni LAMP-positive. The cause of this discrepancy is probably the small number $(n=2)$ of subcultures from a selective agar. As these five specimens were collected from three C. coli culture-positive/C. jejuni culture-negative and two C. jejuni culture-positive/C. coli culture-negative patients, these five patients were actually suspected of having mixed infection with C. jejuni and C. coli. Our assay may reveal mixed infection cases without the need for tedious subculture, and thereby facilitate more accurate epidemiological investigation.

In a preliminary study, $1 \mathrm{ml} 10 \%$ C. jejuni/coli culturepositive faecal homogenate was concentrated by centrifugation to improve the yield of template DNA. The specimen was prepared as described in Methods, and finally $10 \%$ faecal homogenate was concentrated 10 -fold and $5 \mu \mathrm{l}$ was used as the template DNA. The positive rate was, however, decreased, with only 23 of 32 (71.9\%) specimens being positive; 1 of 6 culture-positive/LAMPnegative specimens became LAMP-positive, while 4 of 26 culture-positive/LAMP-positive specimens became LAMPnegative. A possible explanation for this discrepancy may be the influence of inhibitory factors in the stool specimen (Hoshino et al., 1998; Persson \& Olsen, 2005; Ramamurthy et al., 1993), concerns over which were the rationale for our use of $10 \%$ faecal homogenate without concentration.

The frequent occurrence of food poisoning incidents caused by $C$. jejuni/coli, and in particular the recently identified possibility of cases of Guillain-Barré or Fisher syndrome caused by certain strains of C. jejuni (Takahashi et al., 2005), highlight the need for the rapid and accurate identification of these species. Further, the application of our LAMP assay in veterinary and environmental microbiology should 
facilitate a comprehensive approach to the control of human C. jejuni/coli-associated gastroenteritis. Our LAMP assay is a powerful tool for the rapid and simple identification of C. jejuni and C. coli and will facilitate the early diagnosis of food poisoning incidents caused by these organisms.

\section{ACKNOWLEDGEMENTS}

We appreciate the advice and expertise of Dr T. Yoda (Osaka Prefectural Institute of Public Health, Osaka, Japan). In addition, we would like to thank Dr Y. Morikawa (Morikawa Pediatric Clinic, Osaka, Japan), Mr I. Yoneda and Ms K. Fukushima (Suita Municipal Hospital, Osaka, Japan) for providing Campylobacter isolates. This work was supported, in part, by a grant-in-aid from the Daido Life Welfare Foundation and a grant-in-aid from the Ministry of Health, Labour and Welfare (H18-Shokuhin-Ippan-003).

\section{REFERENCES}

Asakura, M., Samosornsuk, W., Taguchi, M., Kobayashi, K., Misawa, N., Kusumotom, M., Nishimura, K., Matsuhisa, A. \& Yamasaki, S. (2007). Comparative analysis of cytolethal distending toxin $(c d t)$ genes among Campylobacter jejuni, C. coli and C. fetus strains. Microb Pathog 42, 174-183.

Fouts, D. E., Mongodin, E. F., Mandrell, R. E., Miller, W. G., Rasko, D. A., Ravel, J., Brinkac, L. M., DeBoy, R. T., Parker, C. T. \& other authors (2005). Major structural differences and novel potential virulence mechanisms from the genomes of multiple Campylobacter species. PLoS Biol 3, e15 (0072-0085).

Furuhata, K., Kakimoto, S., Momoda, T., Kozima, T., Ikedo, M. \& Fukuyama, M. (2006). Comparison of the loop-mediated isothermal amplification (LAMP) method and conventional culture method for the detection of Campylobacter species from retail chickens. Jpn J Food Microbiol 23, 237-241 (in Japanese).

Hara-Kudo, Y., Yoshino, M., Kojima, T. \& Ikedo, M. (2005). Loopmediated isothermal amplification for the rapid detection of Salmonella. FEMS Microbiol Lett 253, 155-161.

Hara-Kudo, Y., Nemoto, J., Ohtsuka, K., Segawa, Y., Takatori, K., Kojima, T. \& Ikedo, M. (2007). Sensitive and rapid detection of vero toxin-producing Escherichia coli using loop-mediated isothermal amplification. J Med Microbiol 56, 398-406.

Hoshino, K., Yamasaki, S., Mukhopadhyay, A. K., Chakraborty, S., Basu, A., Bhattacharya, S. K., Nair, G. B., Shimada, T. \& Takeda, Y. (1998). Development and evaluation of a multiplex PCR assay for rapid detection of toxigenic Vibrio cholerae O1 and O139. FEMS Immunol Med Microbiol 20, 201-207.

Iwamoto, T., Sonobe, T. \& Hayashi, K. (2003). Loop-mediated isothermal amplification for direct detection of Mycobacterium tuberculosis complex, M. avium, and $M$. intracellulare in sputum samples. J Clin Microbiol 41, 2616-2622.

Kulkarni, S. P., Lever, S., Logan, J. M., Lawson, A. J., Stanley, J. \& Shafi, M. S. (2002). Detection of campylobacter species: a comparison of culture and polymerase chain reaction based methods. J Clin Pathol 55, 749-753.

Lawson, A. J., Logan, J. M., O’Neill, G. L., Desai, M. \& Stanley, J. (1999). Large-scale survey of Campylobacter species in human gastroenteritis by PCR and PCR-enzyme-linked immunosorbent assay. J Clin Microbiol 37, 3860-3864.

Linton, D., Owen, R. J. \& Stanley, J. (1996). Rapid identification by PCR of the genus Campylobacter and of five Campylobacter species enteropathogenic for man and animals. Res Microbiol 147, 707-718.
Logan, J. M., Edwards, K. J., Saunders, N. A. \& Stanley, J. (2001). Rapid identification of Campylobacter spp. by melting peak analysis of biprobes in real-time PCR. J Clin Microbiol 39, 2227-2232.

Minami, M., Ohta, M., Ohkura, T., Ando, T., Torii, K., Hasegawa, T. \& Goto, H. (2006). Use of a combination of brushing technique and the loop-mediated isothermal amplification method as a novel, rapid, and safe system for detection of Helicobacter pylori. J Clin Microbiol 44, 4032-4037.

Misawa, N., Kawashima, K., Kawamoto, H. \& Kondo, F. (2002). Development of a combined filtration-enrichment culture followed by a one-step duplex PCR technique for the rapid detection of Campylobacter jejuni and C. coli in human faecal samples. J Med Microbiol 51, 86-89.

Nachamkin, I. (2003). Campylobacter and Arcobacter. In Manual of Clinical Microbiology, vol. 1, 8th edn, pp. 902-914. Edited by P. R. Murray, E. J. Baron, J. H. Jorgensen, M. A. Pfaller \& R. H. Yolken. Washington, DC: American Society for Microbiology.

Nagamine, K., Hase, T. \& Notomi, T. (2002). Accelerated reaction by loop-mediated isothermal amplification using loop primers. Mol Cell Probes 16, 223-229.

Notomi, T., Okayama, H., Masubuchi, H., Yonekawa, T., Watanabe, K., Amino, N. \& Hase, T. (2000). Loop-mediated isothermal amplification of DNA. Nucleic Acids Res 28, E63 (i-vi).

Ohtsuka, K., Yanagawa, K., Takatori, K. \& Hara-Kudo, Y. (2005). Detection of Salmonella enterica in naturally contaminated liquid eggs by loop-mediated isothermal amplification, and characterization of Salmonella isolates. Appl Environ Microbiol 71, 6730-6735.

On, S. L. W. (2005). Taxonomy, phylogeny, and methods for the identification of Campylobacter species. In Campylobacter Molecular and Cellular Biology, pp. 13-42. Edited by J. M. Ketley \& M. E. Konkel. Wymondham, Norfolk: Horizon Bioscience.

Parkhill, J., Wren, B. W., Mungall, K., Ketley, J. M., Churcher, C., Basham, D., Chillingworth, T., Davies, R. M., Feltwell, T. \& other authors (2000). The genome sequence of the food-borne pathogen Campylobacter jejuni reveals hypervariable sequences. Nature 403, 665-668.

Persson, S. \& Olsen, K. E. (2005). Multiplex PCR for identification of Campylobacter coli and Campylobacter jejuni from pure cultures and directly on stool samples. J Med Microbiol 54, 1043-1047.

Ramamurthy, T., Pal, A., Bag, P. K., Bhattacharya, S. K., Nair, G. B., Kurozano, H., Yamasaki, S., Shirai, H., Takeda, T. \& other authors (1993). Detection of cholera toxin gene in stool specimens by polymerase chain reaction: comparison with bead enzyme-linked immunosorbent assay and culture method for laboratory diagnosis of cholera. J Clin Microbiol 31, 3068-3070.

Sails, A. D., Fox, A. J., Bolton, F. J., Wareing, D. R. \& Greenway, D. L. (2003). A real-time PCR assay for the detection of Campylobacter jejuni in foods after enrichment culture. Appl Environ Microbiol 69, 1383-1390.

Shirai, H., Nishibuchi, M., Ramamurthy, T., Bhattacharya, S. K., Pal, S. C. \& Takeda, Y. (1991). Polymerase chain reaction for detection of the cholera enterotoxin operon of Vibrio cholerae. J Clin Microbiol 29, 2517-2521.

Song, T., Toma, C., Nakasone, N. \& Iwanaga, M. (2005). Sensitive and rapid detection of Shigella and enteroinvasive Escherichia coli by a loop-mediated isothermal amplification method. FEMS Microbiol Lett 243, 259-263.

Takahashi, M., Koga, M., Yokoyama, K. \& Yuki, N. (2005). Epidemiology of Campylobacter jejuni isolated from patients with Guillain-Barré and Fisher syndromes in Japan. J Clin Microbiol 43, 335-339. 
Waage, A. S., Vardund, T., Lund, V. \& Kapperud, G. (1999). Detection of small numbers of Campylobacter jejuni and Campylobacter coli cells in environmental water, sewage, and food samples by a seminested PCR assay. Appl Environ Microbiol 65, 1636-1643.

Wang, R. F., Slavic, M. F. \& Cao, W. W. (1992). A rapid PCR method for direct detection of low numbers of Campylobacter jejuni. J Rapid Methods Autom Microbiol 1, 101-108.
Yamazaki-Matsune, W., Taguchi, M., Seto, K., Kawahara, R., Kawatsu, K., Kumeda, Y., Kitazato, M., Nukina, M., Misawa, N. \& Tsukamoto, T. (2007). Development of a multiplex PCR assay for identification of Campylobacter coli, Campylobacter fetus, Campylobacter hyointestinalis subsp. hyointestinalis, Campylobacter jejuni, Campylobacter lari and Campylobacter upsaliensis. J Med Microbiol 56, 1467-1473. 